\title{
Integrated analysis of circular RNA-associated ceRNA network in pancreatic ductal adenocarcinoma
}

\author{
WEI SONG ${ }^{1,2 *}$, WEN-JIE WANG ${ }^{3 *}$, TAO FU $^{1 *}$, LEI CHEN $^{2}$ and DONG-LIU MIAO ${ }^{2}$ \\ ${ }^{1}$ Department of Gastroenterological Surgery II, Renmin Hospital of Wuhan University, Wuhan, \\ Hubei 430060; Departments of ${ }^{2}$ Intervention and Vascular Surgery, and ${ }^{3}$ Radio-Oncology, \\ Affiliated Suzhou Hospital of Nanjing Medical University, Suzhou, Jiangsu 215001, P.R. China
}

Received February 19, 2019; Accepted November 22, 2019

DOI: $10.3892 / \mathrm{ol} .2020 .11306$

\begin{abstract}
Circular RNAs (circRNAs) have displayed dysregulated expression in several types of cancer. However, the functions of the majority of circRNAs in pancreatic ductal adenocarcinoma (PDAC) remain unknown. The present study aimed to investigate the expression, functions and molecular mechanisms of circRNAs in PDAC. The circRNAs, mRNAs and the microRNA (miRNAs) expression profiles were obtained from three Gene Expression Omnibus microarray datasets, and a circRNA-miRNA-mRNA and circRNA-miRNA-hubgene network was established. The interactions between proteins were analyzed using the Search Tool for the Retrieval of Interacting Genes/Proteins database, and hubgenes were identified using the MCODE plugin. A total of eight differentially expressed circRNAs (DEcircRNAs), 44 differentially expressed miRNAs (DEmiRNAs), and 2,052 differentially expressed mRNAs (DEmRNAs) were identified. The present study successfully constructed a circRNA-miRNA-mRNA competing endogenous RNA (ceRNA) network based on four circRNAs, six miRNAs and 111 mRNAs in PDAC. Gene Ontology (GO) and Kyoto Encyclopedia of Genes and Genomes pathways analyses indicated that DEmRNAs may participate in the pathogenesis and progression of PDAC. The protein-protein interaction network and module analysis identified six hubgenes (THBS1, FN1, TIMP3, TGFB2, ITGA1 and ITGA3). Furthermore, the circRNA-miRNA-hubgene regulatory modules were constructed based on the three DEcircRNAs, one DEmiRNAs and five DEmRNAs. In conclusion, the results of the present
\end{abstract}

Correspondence to: Dr Dong-Liu Miao, Department of Intervention and Vascular Surgery, Affiliated Suzhou Hospital of Nanjing Medical University, 16 Baita West Road, Suzhou, Jiangsu 215001, P.R. China E-mail: dongliumiao@126.com

${ }^{*}$ Contributed equally

Key words: pancreatic ductal adenocarcinoma, circular RNA, competitive endogenous RNA, Gene Expression Omnibus study improve the current understanding of the pathogenesis of PDAC.

\section{Introduction}

As the incidence and mortality rates of pancreatic ductal adenocarcinoma (PDAC) continue to increase annually, it has been estimated to become the second leading cause of cancer-associated mortality in Europe and the USA by $2030(1,2)$. To date, surgery is the only treatment option available; however, the 5-year overall survival (OS) time remains unsatisfactory $(1,3)$. This is largely due to a low early diagnostic rate and the fact that the majority of patients exhibit local invasion or distant metastasis (4). In addition, systemic chemotherapy has a limited impact and significant toxicity on the treatment of patients with advanced-stage PDAC. In the majority of cases, these patients are largely resistant to molecularly-targeted agents and immunotherapy (5). Therefore, it is essential to understand the potential mechanism of the carcinogenesis of PDAC and to identify novel markers for developing more effective therapeutic approaches.

Circular RNAs (circRNAs) are a class of noncoding RNAs with continuous and covalently closed circular structures (6). These molecules are not easily degraded by nucleases in the absence of free $3^{\prime}$ and 5 'ends, which makes them more stable than the majority of linear RNAs $(7,8)$. The continuous development of high-throughput sequencing technologies and analysis has allowed for the identification of numerous circRNAs that are abnormally expressed in tumor tissues and have an important influence on tumor progression (9-11). CircRNAs can function as a microRNA (miRNA) sponge to repress miRNA function using miRNA response elements (MREs). This inhibits the activity of miRNAs and regulates the expression of their downstream target genes in numerous types of malignancies (12). Rao et al (13) demonstrated that circRNA-0007874 (circMTO1) expression was decreased in glioblastoma tissues; moreover, elevated circMTO1 expression is known to inhibit cell proliferation and promote apoptosis in both in vivo and in vitro conditions. miR-630 is a targeted miRNA of circMTO1. Therefore, Rao et al (13) established a circMTO1/miR-630/temozolomide (TMZ) competing endogenous RNA (ceRNA) network, 
suggesting that circMTO1 could reverse chemical resistance to TMZ by regulating miR-630. Furthermore, circZFR interacts with C8orf4 through sponging miR-1261 in papillary thyroid carcinoma (14).

In the present study, differentially expressed gene (DEG) expression profiles were obtained from the Gene Expression Omnibus (GEO) database (https://www.ncbi.nlm.nih. gov/geo/). The flowchart for this procedure is presented in Fig. 1. After predicting the sponge miRNA of circRNA and miRNA-mRNA pairs, the present study successfully established the circRNA-miRNA-mRNA network. Subsequently, the present study performed a series of analyses, including functional enrichment analyses and the interactions between proteins. The circRNA-miRNA-hubgene regulatory modules were constructed in order to better understand the pathogenesis of PDAC.

\section{Materials and methods}

Microarray data processing. The present study downloaded two circRNA expression profiles [GSE69362 (15) and GSE79634 (16)] from the GEO database based on the GPL19978 platform (https://www.ncbi.nlm.nih.gov/geo/query/acc.cgi). The present study also downloaded the expression profiles of mRNA and miRNA [GSE60980 (17)] from GEO database based on GPL14550 and GPL15159 platforms (https://www. ncbi.nlm.nih.gov/geo/query/acc.cgi). The GSE69362 dataset included 31 normal pancreatic tissues and six PDAC tissues. The datasets of GSE79634 included 20 PDAC tissues and 20 paracancerous tissues. The array data for GSE60980 included the miRNA expression profiles of 51 PDAC tissues and six normal tissues, and mRNA expression profiles of 49 PDAC tissues and 12 normal tissues. No ethical approval nor informed consent was required in the present study due to the data being publicly available from the GEO.

Screening for DEGs. The raw data from the microarray datasets were normalized and $\log 2$-transformed. The DEGs of each dataset were identified using the Limma package (version 3.40.6) in the Bioconductor package (18). Subsequently, the present study integrated and ranked the differentially expressed circRNAs (DEcircRNAs) with a robust rank aggregation method (19). The FDR $<0.05$ and llog2 fold change (FC) $\mid>1$ were considered as the threshold values for DEGs selection.

Construction of the ceRNA network. The Circular RNA Interactome (https:// circinteractome.nia.nih.gov/) and Cancer-Specific CircRNA databases (http://gb.whu.edu.cn/ $\mathrm{CSCD} /$ ) were used to predict the regulatory relationships between circRNAs and miRNAs. Only the overlapping genes were selected as candidate target miRNAs, which were further screened by the differentially expressed miRNAs (DEmiRNAs). Subsequently, the present study used miRTarBase (20) and TargetScan (21) databases to identify miRNA targeted mRNAs. Only the mRNAs recognized by both databases were considered as candidate mRNAs and were subsequently intersected with the differentially expressed mRNAs (DEmRNAs) in order to determine the DEmRNAs that were targeted by the DEmiRNAs.
The circRNA-miRNA-mRNA network was established using a combination based on circRNA-miRNA pairs and miRNA-mRNA pairs and was visualized using Cytoscape software (version 3.7.0; http://cytoscape.org/).

Functional enrichment analysis. Gene Ontology (GO) and Kyoto Encyclopedia of Genes and Genomes (KEGG) analyses were conducted using the Cluster Profiler package (version 3.12.0) of $\mathrm{R}$ software (version 3.6.1; http://www.r-project.org) (22), in order to assess the primary function of the DEmRNAs in the ceRNA network in tumorigenesis.

Construction of the protein-protein interaction (PPI) network. The present study established a PPI network using the Search Tool for the Retrieval of Interacting Genes (STRING; https://string-db.org/) in order to assess the interactions between DEmRNAs. Cytoscape 3.7.0 was used for visualization. The MCODE plugin was then used to extract hub genes from the PPI network (23).

Statistical analysis. All data were analyzed using $\mathrm{R}$ version 3.6.1. The paired Student's t-test was performed to compare the DEGs between the PDAC tissues and paracancerous tissues and FDR filtering was used for comparative analysis. $\mathrm{P}<0.05$ was considered to indicate a statistically significant difference.

\section{Results}

Identification of differentially expressed circRNA, $m R N A$ and $m i R N A$. In total, 282 and 174 DEcircRNAs were identified from the GSE79634 and GSE69362 datasets, respectively. Among these, 120 upregulated and 162 downregulated circRNAs in the GSE79634 dataset (Fig. 2A), and 116 upregulated and 58 downregulated circRNAs in the GSE69362 dataset (Fig. 2B), were identified. All DEcircRNAs in the GSE79634 and GSE69362 datasets are presented in Fig. 3A. The GSE60980 dataset included mRNA and miRNA expression profiles. Based on this dataset, a total of 44 DEmiRNAs (17 upregulated and 27 downregulated miRNAs) and 2,052 DEmRNAs (1,036 upregulated and 1,016 downregulated mRNAs) were identified in PDAC (Fig. 2C and D). The basic information of the three datasets is listed in Table I. The DEcircRNAs of GSE79634 and GSE69362 datasets were integrated and ranked using a robust method (Table II). In total, eight DEcircRNA (six upregulated and two downregulated circRNAs) were identified $(\mathrm{P}<0.05$; Fig. 3B). The basic characteristics of the eight circRNAs are presented in Table III. The basic structural patterns of the six circRNAs are presented in Fig. 4.

Construction of the ceRNA network. The potential miRNAs targets of the eight DEcircRNAs were retrieved from the CSCD and CircInteractome online database. A total of 409 circRNA-miRNA pairs were identified. After intersecting with the DEmiRNAs, only 10 circRNA-miRNA pairs, including four circRNAs (hsa_circ_0006220, hsa circ_0043278, hsa_circ_0001666 and hsa_circ_0092367) and six DEmiRNAs (hsa-mir-1, hsa-mir-214, hsa-mir-224, 
Table I. Basic information of the three microarray datasets from Gene Expression Omnibus.

\begin{tabular}{lccccccc}
\hline Author & Year & Data source & Platform & Geographical location & Sample size (T/N) & No. of genes & (Refs.) \\
\hline Li et al & 2015 & GSE69362 & GPL19978 & China & $6 / 6$ & 4094 circRNAs & $(15)$ \\
Guo et al & 2016 & GSE79634 & GPL19978 & China & $20 / 20$ & 1836 circRNAs & $(16)$ \\
Sandhu et al & 2015 & GSE60980 & GPL15159 & Norway & $51 / 6$ & 1368 miRNAs & $(17)$ \\
Sandhu et al & 2015 & GSE60980 & GPL14550 & Norway & $49 / 12$ & 42545 mRNAs & $(17)$ \\
\hline
\end{tabular}

circRNAs, circular RNAs; miRNAs, micro RNAs.

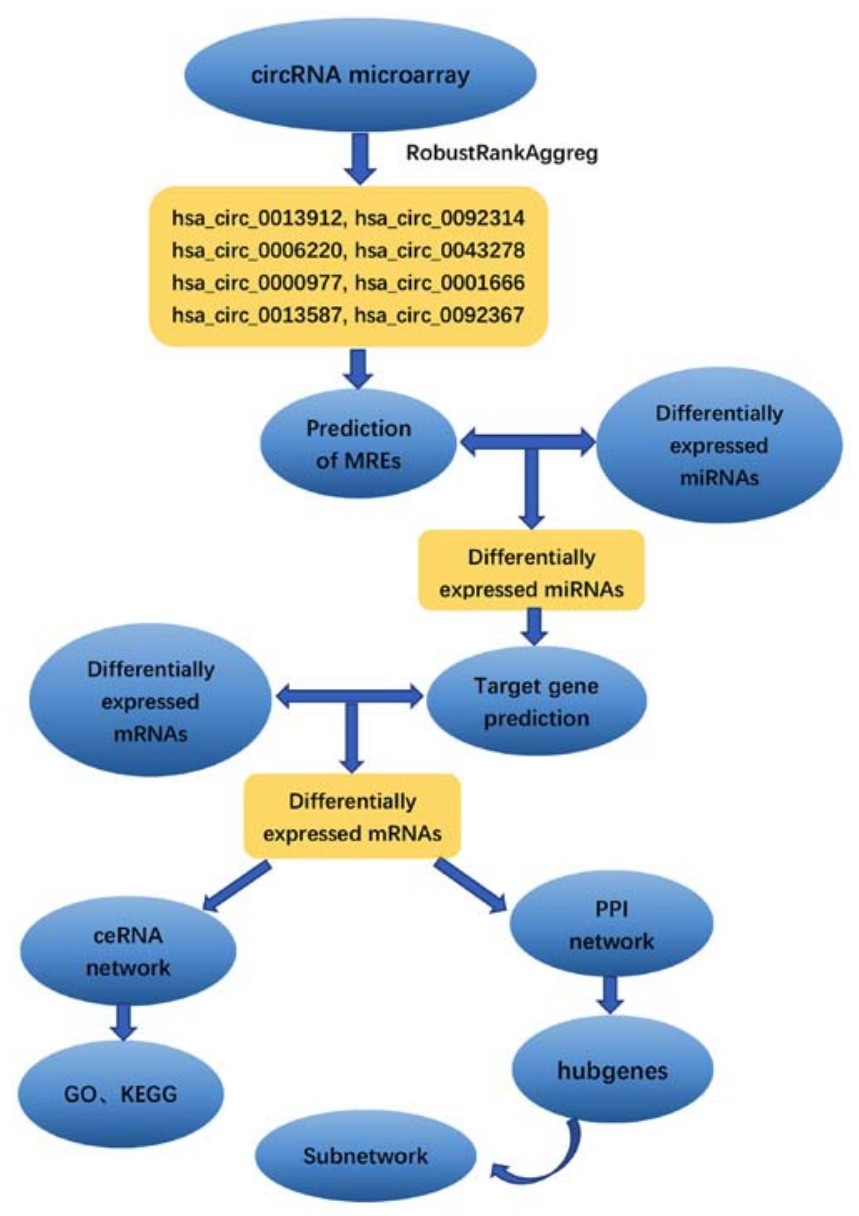

Figure 1. Flowchart of ceRNA network analysis. circRNA, circular RNA; MREs, miRNA response elements; miRNAs, micro RNAs; ceRNA, competing endogenous RNA; PPI network, protein-protein interaction network; GO, gene ontology; KEGG, kyto encyclopedia of genes and genomes.

hsa-mir-223, hsa-mir-1305 and hsa-mir-375), remained. Subsequently, the miRTarBase and TargetScan databases were used to identify target mRNAs of six DEmiRNAs. After the targeted mRNAs intersected with DEmRNAs, the remaining DEmRNAs were used as candidate genes. The results indicate that the ceRNA network included 111 DEmRNAs. Finally, the present study constructed a ceRNA network based on the four circRNAs, six miRNAs and 111 mRNAs (Fig. 5).

Functional enrichment analysis of DEmRNA.GO and KEGG pathways analyses were performed in order to investigate the
Table II. Total of 8 differentially expressed circRNAs using robust rank aggregation method.

\begin{tabular}{llll}
\hline circRNAID & $\operatorname{logFC}$ & P-value & FDR \\
\hline hsa_circ_0013912 & 2.751753 & $1.58 \times 10^{-11}$ & $7.76 \times 10^{-10}$ \\
hsa_circ_0092314 & 2.01195 & $1.66 \times 10^{-10}$ & $4.63 \times 10^{-09}$ \\
hsa_circ_0006220 & 3.736558 & 0.000103 & 0.00307 \\
hsa_circ_0043278 & 3.426752 & 0.000141 & 0.00405 \\
hsa_circ_0000977 & 3.517108 & 0.000201 & 0.00555 \\
hsa_circ_0001666 & 3.124223 & 0.013511 & 0.023253 \\
hsa_circ_0013587 & -2.15798 & $1.51 \times 10^{-09}$ & $2.44 \times 10^{-08}$ \\
hsa_circ_0092367 & -1.81243 & $4.40 \times 10^{-07}$ & $2.72 \times 10^{-06}$ \\
\hline
\end{tabular}

circRNA, circular RNA; FC, fold change; FDR, false discovery rate.

biological function of the 111 DEmRNAs. Among the 129 biological process terms, the most enriched GO terms were 'extracellular matrix organization' and 'extracellular structure organization' $(\mathrm{P}<0.05)$. The mRNAs associated with cellular components were most relevant to the extracellular matrix $(\mathrm{P}<0.05)$. In terms of molecular function, DEmRNAs were primarily enriched in 'cell adhesion molecule binding' $(\mathrm{P}<0.05)$. The GO terms are presented in Table IV. Furthermore, the KEGG pathway analysis indicated that the majority of the DEmRNAs were involved in 'focal adhesion' and 'microRNAs in cancer'. The KEGG pathways are presented in Table V.

Construction of PPI network and module analysis. The PPI network was constructed, and included 37 nodes and 36 edges (Fig. 6A), following the removal of unconnected nodes. The degree, betweenness centrality and key circRNA-miRNA-mRNA regulatory modules were extracted using the MCODE approach (24) from the PPI network in order to investigate the hubgenes in the process of PDAC carcinogenesis. The significant module contained six nodes and 11 edges. The hubgenes included THBS1, FN1, TGFB2, ITGA1, ITGA3 and TIMP3 (Fig. 6B). Subsequently, the present study established a circRNA-miRNA-hubgene subnetwork (Fig. 7), including 16 subnetwork regulatory modules. Since the expression levels of hsa_circ_0092367 and TGFB2 were inconsistent, the hsa_circ_0092367/hsa-mir-375/TGFB2 regulatory module was excluded. 
Table III. Basic characteristics of the eight differently expressed circRNAs.

\begin{tabular}{llcllll}
\hline circRNAID & \multicolumn{1}{c}{ Position } & $\begin{array}{c}\text { Genomic } \\
\text { length }\end{array}$ & Strand & $\begin{array}{c}\text { Best } \\
\text { transcript }\end{array}$ & Gene symbol & Regulation \\
\hline hsa_circ_0013912 & chr1:145601529-145601852 & 323 & Antisense & NM_006468 & POLR3C & Up \\
hsa_circ_0092314 & chr22:20113099-20113439 & 340 & Sense & NM_002882 & RANBP1 & Up \\
hsa_circ_0006220 & chr17:35800605-35800763 & 158 & Sense & NM_001488 & TADA2A & Up \\
hsa_circ_0043278 & chr17:35797838-35800763 & 2925 & Sense & NM_001488 & TADA2A & Up \\
hsa_circ_0000977 & chr2:10784445-10808849 & 24404 & Antisense & NM_024894 & NOL10 & Up \\
hsa_circ_0001666 & chr6:170626457-170639638 & 13181 & Sense & NM_032448 & FAM120B & Up \\
hsa_circ_0013587 & chr1:113661854-113662145 & 291 & Sense & NM_014813 & LRIG2 & Down \\
hsa_circ_0092367 & chr15:25325262-25326442 & 1180 & Sense & NR_003329 & SNORD116-14 & Down \\
\hline
\end{tabular}

circRNA, circular RNA.
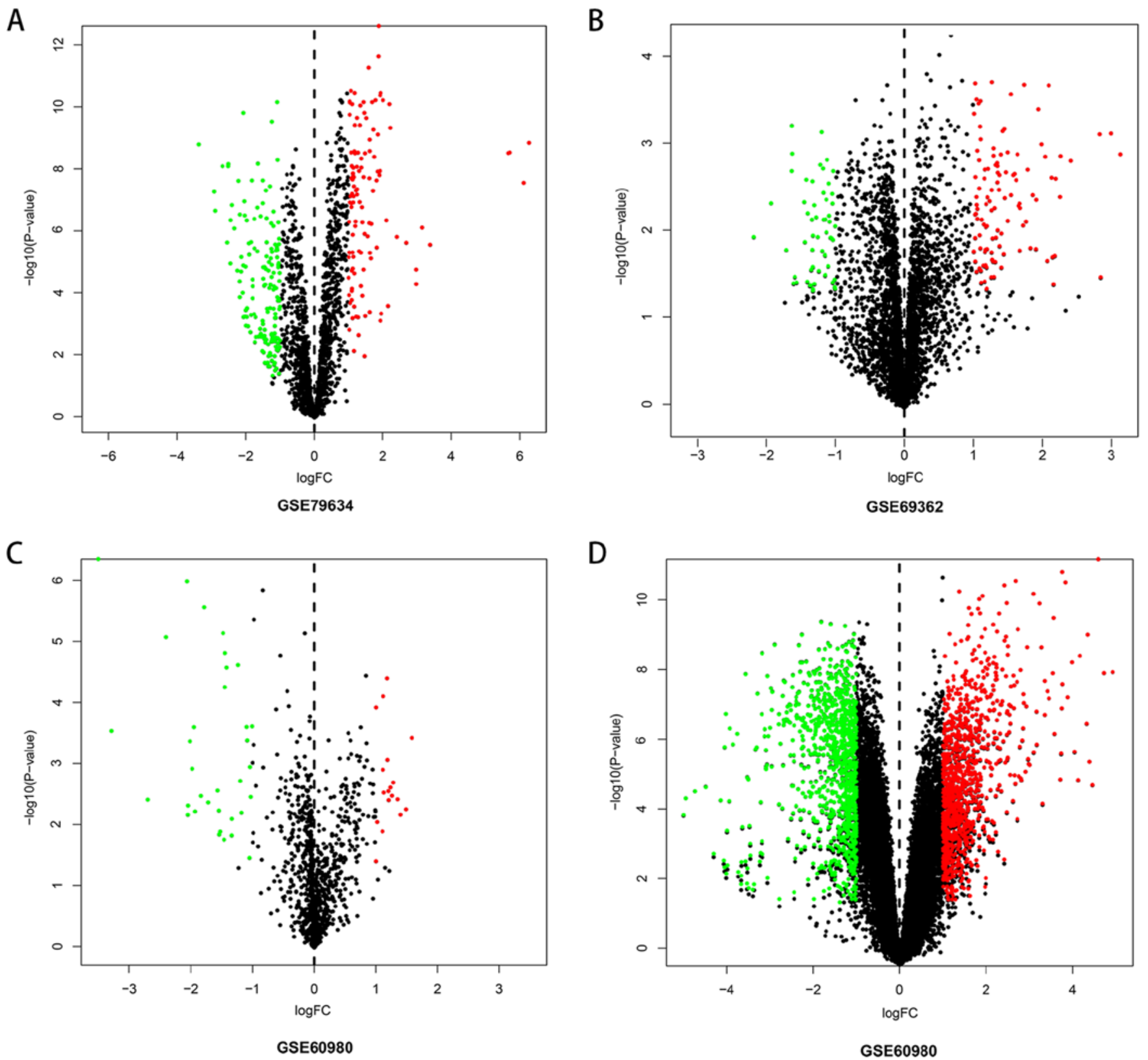

Figure 2. Volcano plot of DEGs of the three microarray datasets. (A) GSE79634 (circRNAs). (B) GSE69362 (circRNAs). (C) GSE60980 (miRNAs). (D) GSE60980 (mRNAs). Red indicates upregulated DEGs and green indicates downregulated DEGs. DEGs, differentially expressed genes. 


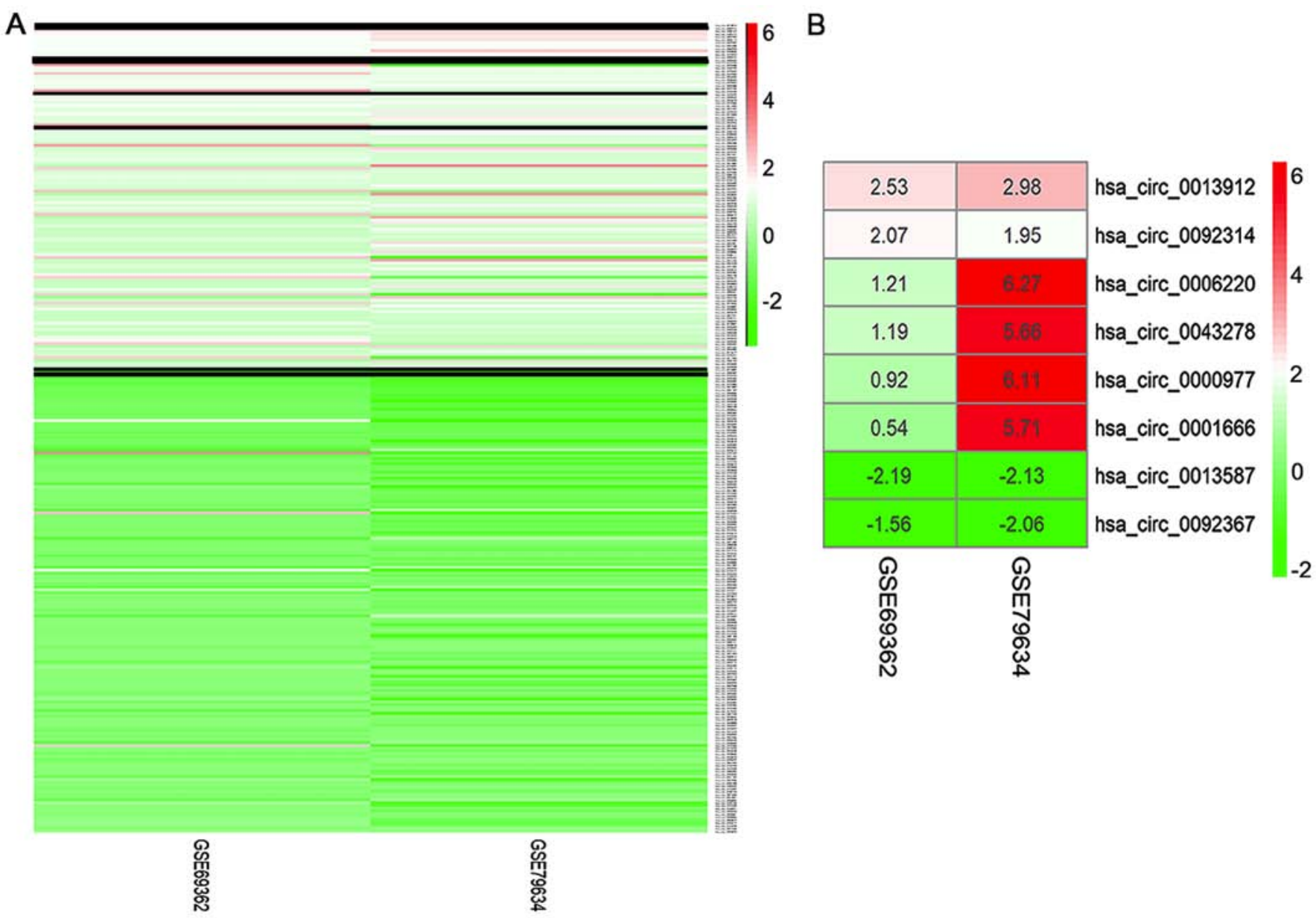

Figure 3. Heatmap of the DEcircRNAs on the GSE79634 and GSE69362 microarray datasets. (A) All DEcircRNAs. (B) Eight DEcircRNAs with robust rank aggregation method. The node color changes gradually from green to red in ascending order according to the $\log 2$ (foldchange) of genes. DEcircRNAs, differentially expressed circRNAs.

\section{Discussion}

circRNAs have become popular topics for research over previous years. circRNAs differ structurally to the well-known linear RNA due to the absence of the $3^{\prime}$ or $5^{\prime}$ polarities or polyadenylated tails (8). This increases their stability and protects them against degradation by the RNase-R enzyme (25). circRNA is abundant in eukaryotic cells where it is found to be structurally stable, highly conserved, and with tissue, timing and disease specificity $(26,27)$. These characteristics make circRNAs potential biomarkers for several types of tumor $(28,29)$. Although the exact functions of the majority of circRNAs remain unclear, a number of recent studies have suggested that circRNAs affect the initiation and development of different types of malignancies (30-32). Cao et al (32) reported that circRNA_100876 was highly expressed in esophageal squamous cell carcinoma. Furthermore, knockdown of circRNA_100876 was demonstrated to inhibit proliferation, migration and progression of the epithelial-mesenchymal transition (EMT). In addition, Chen et al (30) revealed that circPRMT5 promotes the EMT through sponging miR-30c to promote bladder cancer metastasis. Nevertheless, the exact role of circRNAs in PDAC remains undefined.

The present study first performed microarray analysis to identify DEcircRNAs in PDAC samples and associated normal samples. In order to increase the accuracy of the results, the present study used two online databases to predict their MREs. Only the genes that overlapped in both algorithms were selected as candidate miRNAs. The results identified 10 DEcircRNA-DEmiRNA pairs by intersecting with the DEmiRNAs. Using the same technique, the present study identified 120 DEmiRNA-DEmRNA pairs. Subsequently, a circRNA-miRNA-mRNA regulatory network was constructed, including four circRNAs, six miRNAs and 111 mRNAs.

Several studies have indicated that circRNA expression is dysregulated in PDAC, resulting in its pathogenesis and prognosis, and so it is considered to be a tumor-associated biomarker (33-35). Huang et al (34) demonstrated that hsa_ circ_0000977 was significantly upregulated in PDAC tissues. Silencing hsa_circ_0000977 in vitro was revealed to decrease cell proliferation and induce cell cycle arrest. Furthermore, the authors identified hsa_circ_0000977 can regulate the expression of PLK1 by sponging hsa-miR-874-3p in the cytoplasm. Similarly, elevated circ_0030235 was observed in PDAC cell lines. Overexpression of circ_0030235 was associated with low survival rates and advanced clinicopathological features. As such, circ_0030235 is expected to be a prognostic factor for PDAC (35). In the present study, a total of four DEcircRNAs (hsa_circ_0006220, hsa_circ_0043278, hsa_circ_0001666 and hsa_circ_0092367) were identified to be involved in the 
Table IV. GO terms enriched by DEmRNA involved in the ceRNA network.

A, Biological process

\begin{tabular}{llll}
\hline Terms & \multicolumn{1}{c}{ Functional description } & P-value & Gene count \\
\hline GO:0030198 & 'Extracellular matrix organization' & $5.58 \times 10^{-08}$ & 17 \\
GO:0043062 & 'Extracellular structure organization' & $2.77 \times 10^{-07}$ & 17 \\
GO:0048732 & 'Gland development' & $3.19 \times 10^{-05}$ & 15 \\
GO:0001655 & 'Urogenital system development' & $3.19 \times 10^{-05}$ & 13 \\
GO:2000826 & 'Regulation of heart morphogenesis' & $3.19 \times 10^{-05}$ & 6 \\
\hline
\end{tabular}

B, Cellular component

\begin{tabular}{lllc}
\hline Terms & \multicolumn{1}{c}{ Functional description } & P-value & Gene count \\
\hline GO:0031012 & 'Extracellular matrix' & $3.73 \times 10^{-06}$ & 16 \\
GO:0005925 & 'Focal adhesion' & $3.24 \times 10^{-05}$ & 13 \\
GO:0005924 & 'Cell-substrate adherens junction' & $3.24 \times 10^{-05}$ & 13 \\
GO:0030055 & 'Cell-substrate junction' & $3.24 \times 10^{-05}$ & 13 \\
GO:0044420 & 'Extracellular matrix component' & 0.000276 & 7 \\
\hline
\end{tabular}

C, Molecular function

\begin{tabular}{llrr}
\hline Terms & \multicolumn{1}{c}{ Functional description } & P-value & Gene count \\
\hline GO:0001968 & 'Fibronectin binding' & 0.000146 & 5 \\
GO:0050839 & 'Cell adhesion molecule binding' & 0.000275 & 14 \\
GO:0005201 & 'Extracellular matrix structural constituent' & 0.000802 & 6 \\
GO:0019838 & 'Growth factor binding' & 0.001385 & 7 \\
GO:0005518 & 'Collagen binding' & 0.002286 & 5 \\
\hline
\end{tabular}

GO, Gene Ontology.

Table V. Kyoto Encyclopedia of Genes and Genomes pathways enriched by differentially expressed mRNA involved in the competing endogenous RNA network.

\begin{tabular}{|c|c|c|c|c|}
\hline Pathway ID & Functional description & P-value & Genes & Count \\
\hline hsa04510 & Focal adhesion & $2.25 \times 10^{-05}$ & $\begin{array}{l}\text { BIRC3/COL4A1/FN1/ITGA1/ITGA3/LAMA4/ } \\
\text { MET/THBS1/VASP }\end{array}$ & 9 \\
\hline hsa04512 & ECM-receptor interaction & $4.13 \times 10^{-05}$ & COL4A1/FN1/ITGA1/ITGA3/LAMA4/THBS1 & 6 \\
\hline hsa05222 & Small cell lung cancer & $8.41 \times 10^{-05}$ & BIRC3/COL4A1/E2F1/FN1/ITGA3/LAMA4 & 6 \\
\hline hsa05206 & MicroRNAs in cancer & 0.0001 & $\begin{array}{l}\text { E2F1/FSCN1/MET/NOTCH3/PDCD4/PIM1/ } \\
\text { SERPINB5/TGFB2/THBS1/TIMP3 }\end{array}$ & 10 \\
\hline hsa05410 & $\begin{array}{l}\text { Hypertrophic cardiomyopathy } \\
\text { (HCM) }\end{array}$ & 0.00047 & ITGA1/ITGA3/TGFB2/TPM2/TPM4 & 5 \\
\hline hsa05414 & Dilated cardiomyopathy (DCM) & 0.000683 & ITGA1/ITGA3/TGFB2/TPM2/TPM4 & 5 \\
\hline hsa05205 & Proteoglycans in cancer & 0.000961 & $\begin{array}{l}\text { FN1/MET/PDCD4/TGFB2/THBS1/TIMP3/ } \\
\text { TWIST1 }\end{array}$ & 7 \\
\hline hsa04933 & $\begin{array}{l}\text { AGE-RAGE signaling pathway } \\
\text { in diabetic complications }\end{array}$ & 0.001052 & COL4A1/F3/FN1/PIM1/TGFB2 & 5 \\
\hline
\end{tabular}

ceRNA network. To the best of our knowledge, these four circRNAs have not previously been reported.
miRNAs are a large class of endogenous noncoding RNAs, 19-25 nucleotides in length, that are involved in the regulation 

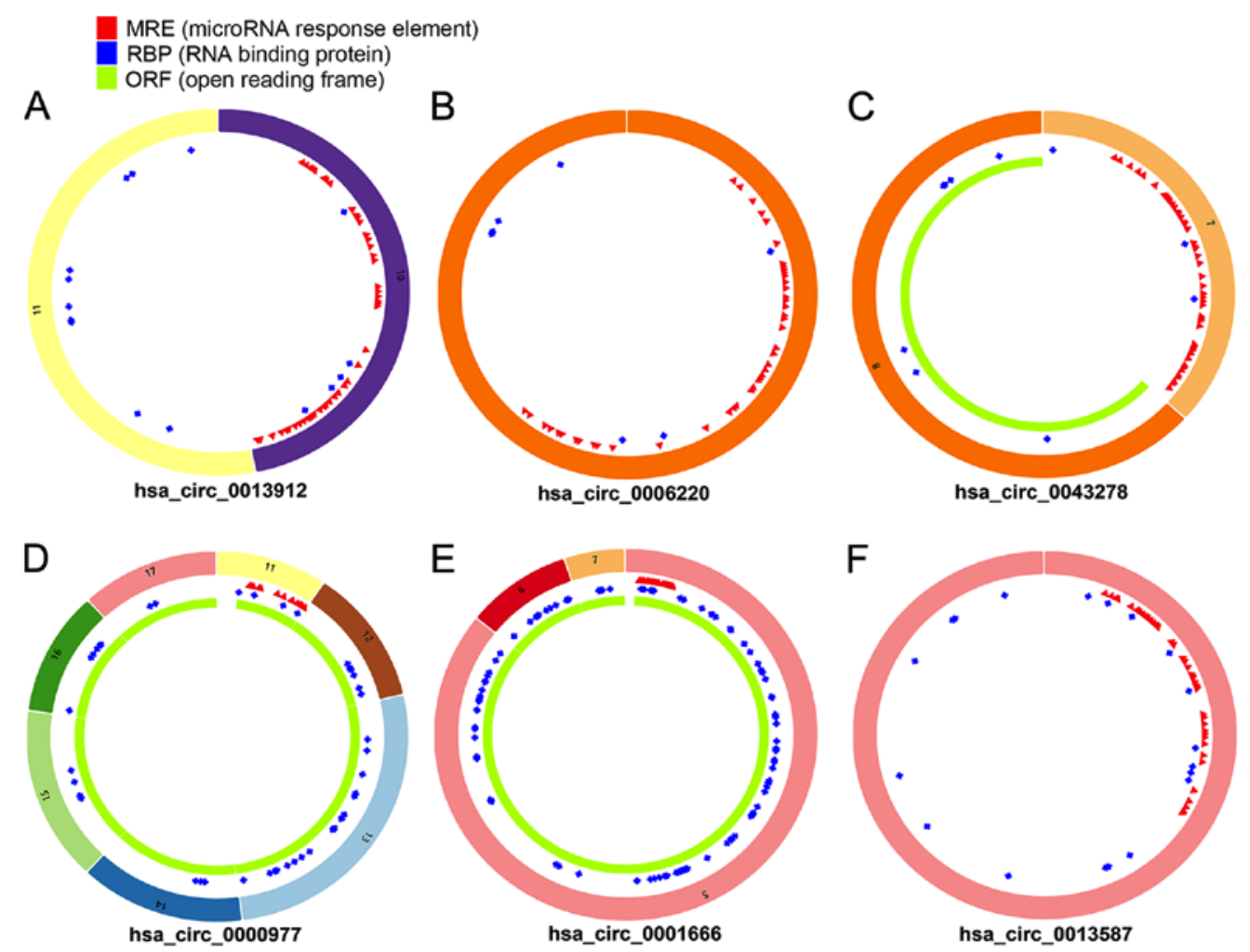

Figure 4. Structural patterns of the seven circRNAs. (A) hsa_circ_0013912, (B) hsa_circ_0006220, (C) hsa_circ_0043278, (D) hsa_circ_0000977, (E) hsa_ circ_0001666, (F) hsa_circ_0013587. Different colors in the circular structure of the circRNA represents the position of the exon.

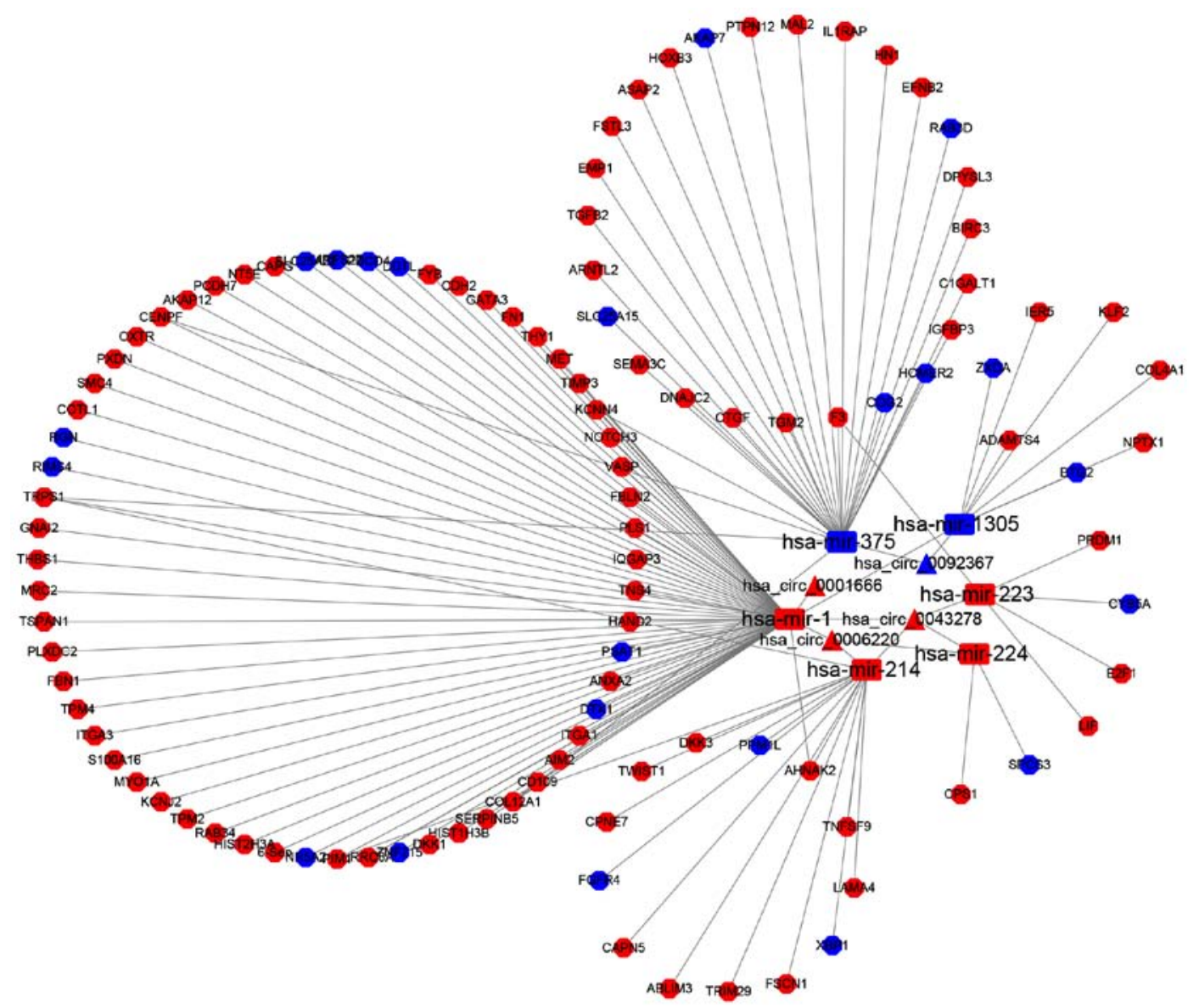

Figure 5. ceRNA network of circRNA-miRNA-mRNA in pancreatic ductal adenocarcinoma. The network consisting of four circRNA nodes, six miRNA nodes, 111 mRNA nodes. Triangles indicate circRNAs, rounded rectangles indicate miRNA, and octagons indicate mRNA. The nodes highlighted in red represent upregulation and the nodes in blue represent downregulation. circRNA, circular RNA; miRNA, micro RNA. 
A

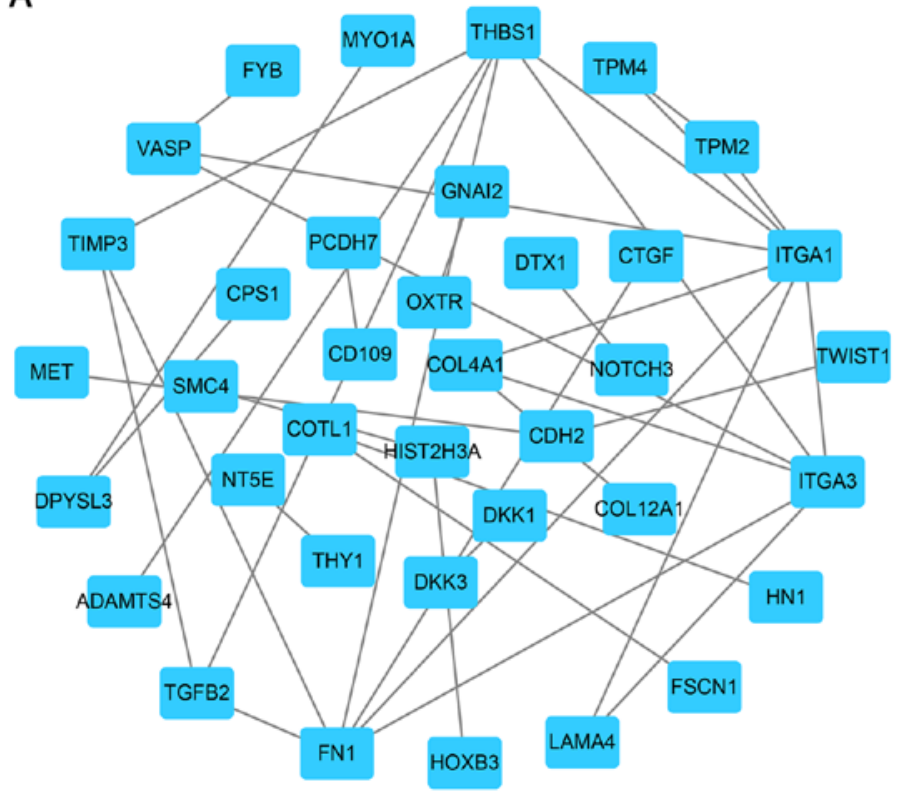

B

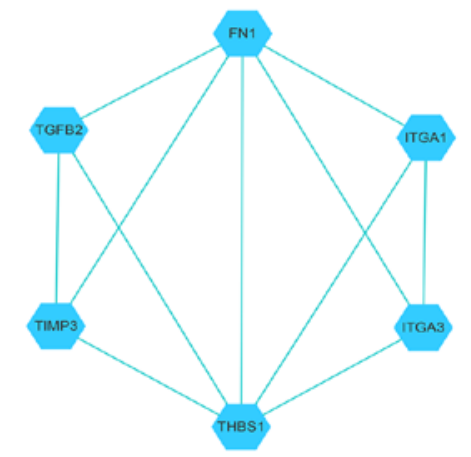

Figure 6. Identification of hubgenes from the PPI network with the MCODE algorithm. This network consists of 37 nodes and 36 edges. (A) The PPI network of 111 genes. (B) The PPI network of six hubgenes that were extracted from (A).

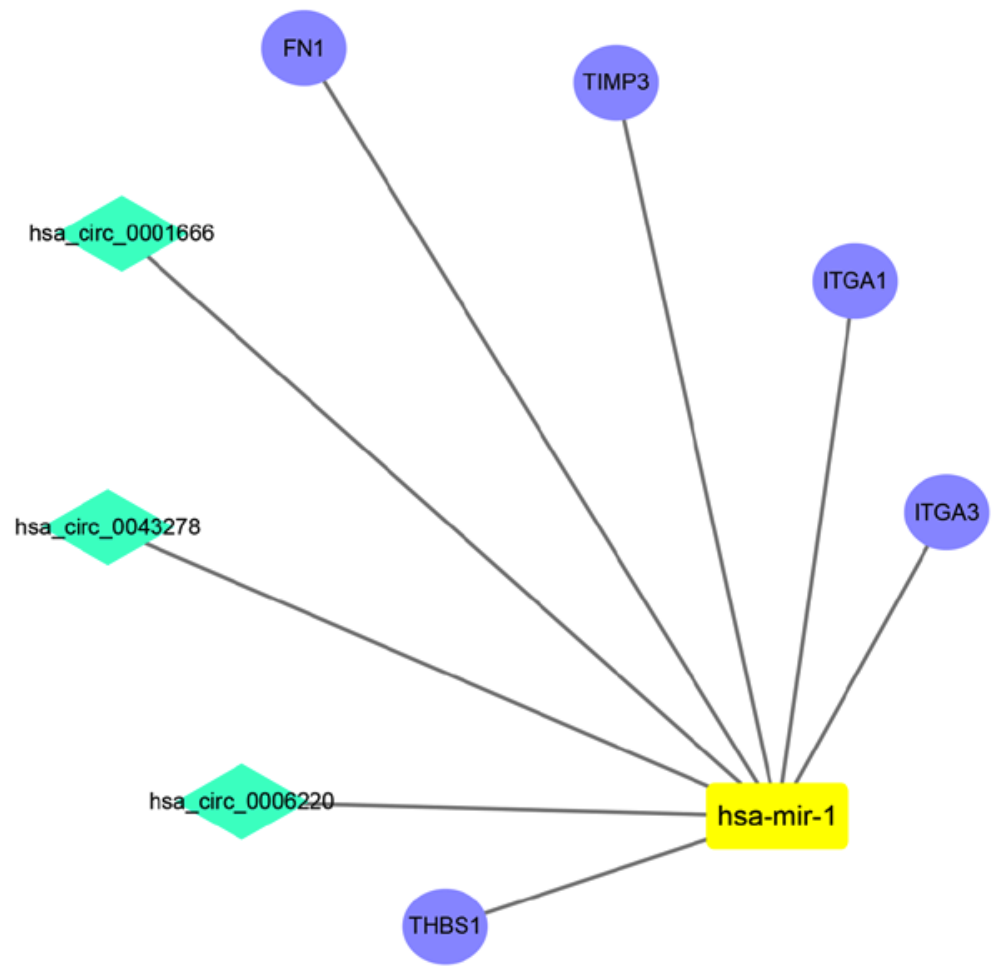

Figure 7. circRNA-miRNA-hubgene network. The network consisting of three circRNAs, one miRNAs, and five hubgenes. Green indicates circRNAs, yellow indicates miRNA, and blue indicates mRNA. Diamonds indicate circRNAs, rounded rectangles indicate miRNA, and ellipses indicate mRNA.

of cell proliferation, differentiation, apoptosis and migration $(36,37)$. Previous studies have investigated the binding of circRNAs to miRNAs and their interactions in pancreatic cancer $(38,39)$. Hao et al $(39)$ indicated that circ_0007534 promoted PDAC cell proliferation, apoptosis and invasion by sponging miR-892b and miR-625. An et al (38) reported that circZMYM2 participates in progression of pancreatic cancer by sponging miR-335-5p. In the present study, we predicted the correlation between four circRNAs and six miRNAs involved in the ceRNA network. Of these six miRNAs, four have previously been reported in PDAC (40-44). Zhu et al (42) demonstrated that miRNA-224 promotes PDAC cell proliferation and migration.

The GO and KEGG enrichment analyses suggest that these DEmRNAs have a significant effect on tumor-associated biological functions. Among the 12 total pathways, 'focal 
adhesion', 'ECM-receptor interaction', 'microRNAs in cancer' and 'proteoglycans in cancer' are associated with the progression of PDAC (45-48). The PPI network was established, including the six hubgenes (THBS1, FN1, TGFB2, ITGA1, ITGA3, and TIMP3), to further identify the key circRNAs participating in the regulatory network. Among these hubgenes, four genes (THBS1, TGFB2, ITGA1 and ITGA3) have been identified to play critical roles in the carcinogenesis and development of PDAC (49-52). However, to the best of our knowledge, the association between these genes and circRNAs has not yet been investigated. The present study established 16 circRNA-miRNA-hubgene axes in PDAC. Since the expression levels of hsa_circ_0092367 and TGFB2 were inconsistent, the hsa_circ_0092367/hsa-mir-375/TGFB2 regulatory module was excluded, leaving 15 circRNA-miRNA-hubgene axes. However, given that the results are based on bioinformatics, further studies are required in order to verify the potential role of the 15 axes in PDAC.

The present study presents several limitations. First, the number of samples is small. Future studies should include larger sample sizes in order to establish more accurate results. As neither of the two GSE datasets used in the present study provided patient survival information, the prognostic value of DEGs was not able to be investigated. In addition, although the patients' clinicopathological parameters were provided in each citation of their dataset, they do not provide the corresponding GEO sample ID number for each patient. Therefore, it was not possible to assess the association between DEGs and the clinicopathological parameters. Furthermore, the conclusions of the present study are only based on the current existing tools and databases, and thus lack in vitro analyses.

The present study successfully established a circRNAassociated ceRNA network and circRNA-miRNA-hubgenes regulatory modules via bioinformatics analysis. The results demonstrated that three significant circRNAs (hsa circ_0006220, hsa_circ_0043278 and hsa_circ_0001666) may play important roles in PDAC progression, which provides new insight into the pathogenesis for patients with PDAC.

\section{Acknowledgements}

Not applicable.

\section{Funding}

The present study was funded by the Suzhou Youth Science and Technology Foundation (grant no. KJXW2018030 and KJXW2018032) and the Suzhou team introduction project (grant no. SZYJTD201712).

\section{Availability of data and materials}

The datasets used and/or analyzed during the current study are available from the corresponding author on reasonable request.

\section{Authors' contributions}

WS and DLM designed the experiment. WJW, TF and LC undertook the data acquisition. WS, TF, and WJW were involved in the interpretation of data. WS and DLM analyzed and visualized the data. All authors drafted and revised the manuscript. The final manuscript was read and approved by all authors.

\section{Ethics approval and consent to participate}

Not applicable.

\section{Patient consent for publication}

Not applicable.

\section{Competing interests}

The authors declare that they have no competing interests.

\section{References}

1. Siegel RL, Miller KD and Jemal A: Cancer statistics, 2015. CA Cancer J Clin 65: 5-29, 2015.

2. Siegel RL, Miller KD and Jemal A: Cancer statistics, 2018. CA Cancer J Clin 68: 7-30, 2018.

3. DeSantis CE, Lin CC, Mariotto AB, Siegel RL, Stein KD, Kramer JL, Alteri R, Robbins AS and Jemal A: Cancer treatment and survivorship statistics, 2014. CA Cancer J Clin 64: 252-271, 2014.

4. Hidalgo M: Pancreatic cancer. N Engl J Med 362: 1605-1617, 2010

5. Chrystoja CC, Diamandis EP, Brand R, Ruckert F, Haun R and Molina R: Pancreatic cancer. Clin Chem 59: 41-46, 2013.

6. Du WW, Zhang C, Yang W, Yong T, Awan FM and Yang BB: Identifying and characterizing circRNA-protein interaction. Theranostics 7: 4183-4191, 2017.

7. Liu J, Liu T, Wang X and He A: Circles reshaping the RNA world: From waste to treasure. Mol Cancer 16: 58, 2017.

8. Li J, Yang J, Zhou P, Le Y, Zhou C, Wang S, Xu D, Lin HK and Gong Z: Circular RNAs in cancer: Novel insights into origins, properties, functions and implications. Am J Cancer Res 5: 472-480, 2015

9. Lv C, Sun L, Guo Z, Li H, Kong D, Xu B, Lin L, Liu T, Guo D, Zhou J and Li Y: Circular RNA regulatory network reveals cell-cell crosstalk in acute myeloid leukemia extramedullary infiltration. J Transl Med 16: 361, 2018.

10. Zhang H, Deng T, Ge S, Liu Y, Bai M, Zhu K, Fan Q, Li J, Ning T, Tian $\mathrm{F}$, et al: Exosome circRNA secreted from adipocytes promotes the growth of hepatocellular carcinoma by targeting deubiquitination-related USP7. Oncogene 38: 2844-2859, 2019.

11. Chen N, Zhao G, Yan X, Lv Z, Yin H, Zhang S, Song W, Li X, Li L, Du Z, et al: A novel FLI1 exonic circular RNA promotes metastasis in breast cancer by coordinately regulating TET1 and DNMT1. Genome Biol 19: 218, 2018.

12. Zhong Y, Du Y, Yang X, Mo Y, Fan C, Xiong F, Ren D, Ye X, Li C, Wang Y, et al: Circular RNAs function as ceRNAs to regulate and control human cancer progression. Mol Cancer 17: 79, 2018.

13. Retraction: Circular RNA-0007874 (circMTO1) reverses chemoresistance to temozolomide by acting as a sponge of microRNA-630 in glioblastoma by Jiang Rao, Xinxin Cheng, Huimin Zhu, Lifeng Wang, Li Liu. Cell Biol Int, Dec 11, 2018 (Epub ahead of print).

14. Wei H, Pan L, Tao D and Li R: Circular RNA circZFR contributes to papillary thyroid cancer cell proliferation and invasion by sponging miR-1261 and facilitating C8orf4 expression. Biochem Biophys Res Commun 503: 56-61, 2018.

15. Li H, Hao X, Wang H, Liu Z, He Y, Pu M, Zhang H, Yu H, Duan J and Qu S: Circular RNA expression profile of pancreatic ductal adenocarcinoma revealed by microarray. Cell Physiol Biochem 40: 1334-1344, 2016.

16. Guo S, Xu X, Ouyang Y, Wang Y, Yang J, Yin L, Ge J and Wang H: Microarray expression profile analysis of circular RNAs in pancreatic cancer. Mol Med Rep 17: 7661-7671, 2018.

17. Sandhu V, Bowitz Lothe IM, Labori KJ, Skrede ML, Hamfjord J, Dalsgaard AM, Buanes T, Dube G, Kale MM, Sawant S, et al: Differential expression of miRNAs in pancreatobiliary type of periampullary adenocarcinoma and its associated stroma. Mol Oncol 10: 303-316, 2016. 
18. Ritchie ME, Phipson B, Wu D, Hu Y, Law CW, Shi W and Smyth GK: limma powers differential expression analyses for RNA-sequencing and microarray studies. Nucleic Acids Res 43: e47, 2015.

19. Kolde R, Laur S, Adler P and Vilo J: Robust rank aggregation for gene list integration and meta-analysis. Bioinformatics 28: 573-580, 2012.

20. Chou CH, Shrestha S, Yang CD, Chang NW, Lin YL, Liao KW, Huang WC, Sun TH, Tu SJ, Lee WH, et al: miRTarBase update 2018: A resource for experimentally validated microRNA-target interactions. Nucleic Acids Res 46 (D1): D296-D302, 2018.

21. Agarwal V, Bell GW, Nam JW and Bartel DP: Predicting effective microRNA target sites in mammalian mRNAs. eLife 4: e05005 2015

22. Yu G, Wang LG, Han Y and He QY: clusterProfiler: An R package for comparing biological themes among gene clusters. OMICS 16: 284-287, 2012.

23. Bandettini WP, Kellman P, Mancini C, Booker OJ, Vasu S, Leung SW, Wilson JR, Shanbhag SM, Chen MY and Arai AE: MultiContrast delayed enhancement (MCODE) improves detection of subendocardial myocardial infarction by late gadolinium enhancement cardiovascular magnetic resonance: A clinical validation study. J Cardiovasc Magn Reson 14: 83, 2012.

24. Bader GD and Hogue CW: An automated method for finding molecular complexes in large protein interaction networks. BMC Bioinformatics 4: 2, 2003.

25. Zhang Y, Zhang XO, Chen T, Xiang JF, Yin QF, Xing YH, Zhu S, Yang L and Chen LL: Circular intronic long noncoding RNAs. Mol Cell 51: 792-806, 2013.

26. Jeck WR, Sorrentino JA, Wang K, Slevin MK, Burd CE, Liu J, Marzluff WF and Sharpless NE: Circular RNAs are abundant, conserved, and associated with ALU repeats. RNA 19: 141-157, 2013

27. Rybak-Wolf A, Stottmeister C, Glažar P, Jens M, Pino N, Giusti S, Hanan M, Behm M, Bartok O, Ashwal-Fluss R, et al: Circular RNAs in the mammalian brain are highly abundant, conserved, and dynamically expressed. Mol Cell 58: 870-885, 2015.

28. Meng S, Zhou H,Feng Z, Xu Z, Tang Y,Li P and Wu M: CircRNA: Functions and properties of a novel potential biomarker for cancer. Mol Cancer 16: 94, 2017

29. Jiang XM, Li ZL, Li JL, Xu Y, Leng KM, Cui YF and Sun DJ: A novel prognostic biomarker for cholangiocarcinoma: circRNA Cdrlas. Eur Rev Med Pharmacol Sci 22: 365-371, 2018.

30. Chen X, Chen RX, Wei WS, Li YH, Feng ZH, Tan L, Chen JW, Yuan GJ, Chen SL, Guo SJ, et al: PRMT5 circular RNA promotes metastasis of urothelial carcinoma of the bladder through sponging miR-30c to induce epithelial-mesenchymal transition. Clin Cancer Res 24: 6319-6330, 2018.

31. Liu X, Abraham JM, Cheng Y, Wang Z, Wang Z, Zhang G, Ashktorab H, Smoot DT, Cole RN, Boronina TN, et al: Synthetic Circular RNA functions as a miR-21 sponge to suppress gastric carcinoma cell proliferation. Mol Ther Nucleic Acids 13: 312-321, 2018.

32. Cao S, Chen G, Yan L, Li L and Huang X: Contribution of dysregulated circRNA_100876 to proliferation and metastasis of esophageal squamous cell carcinoma. Onco Targets Ther 11: 7385-7394, 2018 .

33. Yang F, Liu DY, Guo JT, Ge N, Zhu P, Liu X, Wang S, Wang GX and Sun SY: Circular RNA circ-LDLRAD3 as a biomarker in diagnosis of pancreatic cancer. World J Gastroenterol 23: 8345-8354, 2017

34. Huang WJ, Wang Y, Liu S, Yang J, Guo SX, Wang L, Wang H and Fan YF: Silencing circular RNA hsa_circ_0000977 suppresses pancreatic ductal adenocarcinoma progression by stimulating miR-874-3p and inhibiting PLK1 expression. Cancer Lett 422: 70-80, 2018.

35. Xu Y, Yao Y, Gao P and Cui Y: Upregulated circular RNA circ 0030235 predicts unfavorable prognosis in pancreatic ductal adenocarcinoma and facilitates cell progression by sponging miR-1253 and miR-1294. Biochem Biophys Res Commun 509: 138-142, 2019.

36. Bartel DP: MicroRNAs: Target recognition and regulatory functions. Cell 136: 215-233, 2009.
37. Liu C, Liu R, Zhang D, Deng Q, Liu B, Chao HP, Rycaj K, Takata Y, Lin K, Lu Y, et al: MicroRNA-141 suppresses prostate cancer stem cells and metastasis by targeting a cohort of pro-metastasis genes. Nat Commun 8: 14270, 2017.

38. An Y, Cai H, Zhang Y, Liu S, Duan Y, Sun D, Chen X and He X: circZMYM2 competed endogenously with miR-335-5p to regulate JMJD2C in pancreatic cancer. Cell Physiol Biochem 51: 2224-2236, 2018.

39. Hao L, Rong W, Bai L, Cui H, Zhang S, Li Y, Chen D and Meng X: Upregulated circular RNA circ_0007534 indicates an unfavorable prognosis in pancreatic ductal adenocarcinoma and regulates cell proliferation, apoptosis, and invasion by sponging miR-625 and miR-892b. J Cell Biochem 120: 3780-3789, 2019.

40. Cheng Q, Han LH, Zhao HJ, Li H and Li JB: Abnormal alterations of miR-1 and miR-214 are associated with clinicopathological features and prognosis of patients with PDAC. Oncol Lett 14: 4605-4612, 2017.

41. Cao TH, Ling X, Chen C, Tang W, Hu DM and Yin GJ: Role of miR-214-5p in the migration and invasion of pancreatic cancer cells. Eur Rev Med Pharmacol Sci 22: 7214-7221, 2018.

42. Zhu G, Zhou L, Liu H, Shan Y and Zhang X: MicroRNA-224 promotes pancreatic cancer cell proliferation and migration by targeting the TXNIP-mediated HIF1 $\alpha$ pathway. Cell Physiol Biochem 48: 1735-1746, 2018.

43. He D, Huang C, Zhou Q, Liu D, Xiong L, Xiang H, Ma G and Zhang Z: HnRNPK/miR-223/FBXW7 feedback cascade promotes pancreatic cancer cell growth and invasion. Oncotarget 8: 20165-20178, 2017.

44. Yang D, Yan R, Zhang X, Zhu Z, Wang C, Liang C and Zhang X: Deregulation of MicroRNA-375 inhibits cancer proliferation migration and chemosensitivity in pancreatic cancer through the association of HOXB3. Am J Transl Res 8: 1551-1559, 2016.

45. Begum A, Ewachiw T, Jung C, Huang A, Norberg KJ, Marchionni L, McMillan R, Penchev V, Rajeshkumar NV, Maitra A, et al: The extracellular matrix and focal adhesion kinase signaling regulate cancer stem cell function in pancreatic ductal adenocarcinoma. PLoS One 12: e0180181, 2017.

46. Wang J, Dumartin L, Mafficini A, Ulug P, Sangaralingam A Alamiry NA, Radon TP, Salvia R, Lawlor RT, Lemoine NR, et al: Splice variants as novel targets in pancreatic ductal adenocarcinoma. Sci Rep 7: 2980, 2017.

47. Dhayat SA, Traeger MM, Rehkaemper J, Stroese AJ, Steinestel K, Wardelmann E, Kabar I and Senninger N: Clinical impact of epithelial-to-mesenchymal transition regulating MicroRNAs in pancreatic ductal adenocarcinoma. Cancers (Basel) 10: pii: E328, 2018.

48. Kumar S, Inigo JR, Kumar R, Chaudhary AK, O'Malley J, Balachandar S, Wang J, Attwood K, Yadav N, Hochwald S, et al: Nimbolide reduces CD44 positive cell population and induces mitochondrial apoptosis in pancreatic cancer cells. Cancer Lett 413: 82-93, 2018.

49. Asama H, Suzuki R, Hikichi T, Takagi T, Masamune A and Ohira H: MicroRNA let-7d targets thrombospondin-1 and inhibits the activation of human pancreatic stellate cells. Pancreatology 19: 196-203, 2019.

50. D'Cruz OJ, Qazi S, Hwang L, Ng K and Trieu V: Impact of targeting transforming growth factor $\beta-2$ with antisense OT-101 on the cytokine and chemokine profile in patients with advanced pancreatic cancer. Onco Targets Ther 11: 2779-2796, 2018.

51. Gharibi A, La Kim S, Molnar J, Brambilla D, Adamian Y, Hoover M, Hong J, Lin J, Wolfenden L and Kelber JA: ITGA1 is a pre-malignant biomarker that promotes therapy resistance and metastatic potential in pancreatic cancer. Sci Rep 7: 10060, 2017.

52. Idichi T, Seki N, Kurahara H, Fukuhisa H, Toda H, Shimonosono M, Yamada Y, Arai T, Kita Y, Kijima Y, et al: Involvement of anti-tumor miR-124-3p and its targets in the pathogenesis of pancreatic ductal adenocarcinoma: Direct regulation of ITGA3 and ITGB1 by miR-124-3p. Oncotarget 9: 28849-28865, 2018.

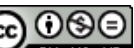

This work is licensed under a Creative Commons Attribution-NonCommercial-NoDerivatives 4.0 International (CC BY-NC-ND 4.0) License. 